\title{
荒漠绿洲过渡带植被空间异质性的 可塑性面积单元问题
}

\author{
何志斌 ${ }^{1}$ 赵文智 ${ }^{*}$ 常学礼 ${ }^{2}$ \\ （1 中国科学院寒区旱区环境与工程研究所，兰州 730000）（2 烟台师范大学地理旅游系, 山东烟台 264025)
}

摘 要 以荒漠绿洲过渡带植被为研究对象, 采用地统计理论与方法, 以优势植物泡泡刺 ( Nitraria sphaerocarpa) 和 红砂 (Reaumuria soongorica) 种群为例 采用一个样地内 2500 个 $10 \mathrm{~m} \times 10 \mathrm{~m}$ 小样方中获取的植物盖度数据来研究采 样尺度和划区方式对荒漠植被空间异质性的影响。其中尺度效应研究的思路是 :一是保持粒度 (样方)不变, 增加 幅度(样地)分析其对空间异质性的影响：二是保持幅度不变, 改变粒度进行分析。划区效应是在样地尺度 $(500 \mathrm{~m} \times$ $500 \mathrm{~m})$ 上, 把整块样地数据聚合成 $10 \mathrm{~m} \times 200 \mathrm{~m} 、 20 \mathrm{~m} \times 100 \mathrm{~m} 、 40 \mathrm{~m} \times 50 \mathrm{~m} 、 50 \mathrm{~m} \times 40 \mathrm{~m} 、 100 \mathrm{~m} \times 20 \mathrm{~m}$ 和 $200 \mathrm{~m} \times 10 \mathrm{~m}$ 等不同形状和不同方向的网格进行地统计分析。结果表明 : 荒漠绿洲过渡带植被空间异质性受采样尺度和划区方 式影响显著。当样地面积小于 $200 \mathrm{~m} \times 200 \mathrm{~m}$ 时, 所获取的数据不能真实反映泡泡刺种群的空间异质性; 当样地面 积小于 $100 \mathrm{~m} \times 100 \mathrm{~m}$ 时, 所获取的数据不能真实反映红砂种群的空间异质性。调查泡泡刺和红砂种群的空间格局 时 样方面积在 $20 \mathrm{~m} \times 20 \mathrm{~m}$ 到 $30 \mathrm{~m} \times 30 \mathrm{~m}$ 较为合适。植被空间异质性参数对划区方式的敏感性较强, 其中泡泡刺 种群的敏感性高于红砂种群。

关键词 荒漠绿洲过渡带 空间异质性 可塑性面积单元 尺度效应 划区效应

\section{THE MODIFIABLE AREAL UNIT PROBLEM OF SPATIAL HETEROGENEITY OF VEGETATION COMMUNITIES IN THE TRANSITIONAL ZONE BETWEEN OASIS AND DESERT}

\author{
HE Zhi-Bin ${ }^{1}$ ZHAO Wen-Zhi ${ }^{*}$ and CHANG Xue-Li ${ }^{2}$ \\ (1 Cold and Arid Regions Environmental and Engineering Research Institute , Chinese Academy of Sciences , Lanzhou 730000 , China) \\ (2 Department of Geography and Tourism, Yantai Normal University, Yantai, Shandong 264025 , China)
}

\begin{abstract}
The modifiable areal unit problem has significant implications for ecological research that involves the use of spatial data. The objective of the present study was to discuss the scale effect and zoning effect by investigating and analyzing the spatial heterogeneity of vegetation communities in the transitional zone between oasis and deserts. Geostatistical techniques were used to evaluate the spatial characteristics of plant communities. The spatial structures of different plant populations were characterized using exponential model variogram parameters , including nugget $\left(C_{0}\right)$, range $\left(A_{0}\right)$ and sill $\left(C_{0}+C\right)$. Two methods were employed to determine the scale effect of spatial heterogeneity :1) a constant plot area $(10 \mathrm{~m} \times 10 \mathrm{~m})$ and the sampling area expanded to analyze spatial heterogeneity of plant populations ;2) the plot area was changed for maintaining a constant sampling area to analyze spatial heterogeneity. In addition , the plots at the scale of $500 \mathrm{~m} \times 500 \mathrm{~m}$ were clustered into nested units of different shapes and directions $(10 \mathrm{~m} \times 200 \mathrm{~m}, 20 \mathrm{~m} \times 100 \mathrm{~m}, 40 \mathrm{~m} \times 50 \mathrm{~m}, 50 \mathrm{~m}$ $\times 40 \mathrm{~m}, 100 \mathrm{~m} \times 20 \mathrm{~m}$ and $200 \mathrm{~m} \times 10 \mathrm{~m}$ ) and analyzed at a resolution of $10 \mathrm{~m} \times 10 \mathrm{~m}$ to examine the zoning effect. The results showed that the spatial heterogeneity of plant populations in the transitional zone between oasis and desert were influenced significantly by scale and zoning approach. Using sensitivity analysis, we were able to obtain a suitable sampling area and zoning approach to weaken the scale and zoning effects. The scale at which different parameters were sensitive was variable. For example, $C_{0}$ and $C_{0}+C$ were relatively sensitive at small scales, $A_{0}$ was affected significantly by changes at larger scales, and $C_{0}$ and $A_{0}$ were relatively sensitive to different zoning approaches. In order to gain more representative vegetation information of spatial heterogeneity, the sampling area should be more than $200 \mathrm{~m} \times 200 \mathrm{~m}$ for Nitraria sphaerocarpa populations , $100 \mathrm{~m} \times 100 \mathrm{~m}$ for Reaumuria soongorica populations, and a plot area of $20 \mathrm{~m} \times 20 \mathrm{~m}$ to $30 \mathrm{~m} \times 30 \mathrm{~m}$ should be feasible.
\end{abstract}


Key words Transitional zone between oasis and desert, Spatial heterogeneity, The modifiable areal unit problem , Scale effect , Zoning effect

如何获取有关我们生存星球复杂的生态现象和 生态问题的信息以及如何利用这些信息解决它们变 得越来越重要 (Gallagher et al. ,1995)。在生态学研 究中 , 许多信息(数据) 都与面积相联系。在分析这 些数据时, 常常出现其结果随面积单元(栅格细胞或 粒度) 定义的不同而发生变化, 即所谓的可塑性面积 单元问题(邬建国 2000), ,它包括尺度效应和划区效 应两个方面, 其中尺度问题最具复杂性和多样性。 若要正确理解植被格局与过程的关系, 就必须深入 了解其尺度依赖的特点, 即尺度效应 (Scale effect) (邬建国，1996）。在同一尺度上会有很多种划区方 案(划区方向和划区形状)，而且不同的划区方案会 引起分析结果的变化, 即划区效应 (Zoning effect)。 因此, 可塑性单元问题已成为地理学和生态学研究 的重要问题 (Turner, 1989a; Dudley, 1991 ; Levin, 1992)。

Gehlke 和 Biehl(1934) 在统计学中最早注意到尺 度效应, Yule 和 Kendall(1950)进一步阐述了尺度效 应对统计结果的影响, 指出统计分析结果只对所采 用的面积单元有效，而在其它尺度上不一定有效。 近年来, 可塑性面积单元问题的研究更加受到重视, 例如 Openshaw 和 Taylor (1979) 在一项相关分析研究 中表明,在改变尺度(面积单元大小)和划区方法时， 相关系数可以是取值范围 $(-1 \sim 1)$ 内的任何值。随 着景观生态学和等级理论的兴起, 许多生态学研究 注意到了尺度改变对格局和过程分析结果的影响 (Turner , 1989b ; Jenerette \& Wu , 2000 ; Wu \& Qi , 2000) ,也注意到如何解决可塑性面积单元问题 ( Hay et al. ,2003)。

在国内这方面的研究也较多, 例如朱锦禁等 (1999)研究了森林群落物种多样性的可塑性面积单 元问题, 王政权等 (2000) 和李友常等 (1997) 分别研 究了红松老龄林主要树种和天牛种群的尺度依赖问 题, 陈玉福等 (2003)论述了生态系统的空间异质性, 并讨论了空间异质性的尺度含义。这些研究在理论 上推进了国内相关研究的进程, 在实践上对研究区 域生态格局和生态过程的认识和生态环境建设布局 起到重要指导作用。

荒漠植被结构相对简单, 物种组成相对较少, 而 且多呈斑块状不连续分布格局。有关斑块的形状和 大小以及斑块内部的生态过程和生态格局的研究相
对薄弱。荒漠植被特别是荒漠绿洲过渡带斑块状的 植被格局对维护荒漠绿洲的生态安全起着重要作 用。因此, 探索荒漠绿洲过渡带天然斑块状植被的 格局和过程已经成为模拟天然植被来建立人工防护 体系, 提高防护功能和延续防护时间的需求。但是 目前的研究对采样尺度和划区方式都是凭经验而 定，具有一定的随意性，忽视了植被空间异质性的尺 度效应和划区效应。鉴于此, 本文以荒漠绿洲过渡 带泡泡刺 ( Nitraria sphaerocarpa) 和红砂 (Reaumuria soongorica) 种群为研究对象，探讨植被空间数据获取 的可塑性单元问题, 旨在为研究认识荒漠植被空间 异质性的尺度效应和划区效应提供指导。

\section{1 研究区概况与研究方法}

\section{1 研究区概况}

研究区选在黑河中游甘肃省临泽县北部绿洲边 缘地理坐标 $\left(39^{\circ} 20^{\prime} \mathrm{N} ; 100^{\circ} 08^{\prime} \mathrm{E}\right)$ ，处于荒漠、巴丹 吉林沙漠和张临高绿洲的交汇处，样地的南侧和东 侧为绿洲, 分别相距 $0.5 \mathrm{~km}$ 和 $1.5 \mathrm{~km}$, 西侧 $2 \mathrm{~km}$ 以 外为祁连山余脉浅山区，有时可形成径流 北侧为荒 漠戈壁和沙漠(巴丹吉林沙漠延伸带) 地势平坦, 海 拔 $1350 \mathrm{~m}$ 左右, 年平均降水量 $117.1 \mathrm{~mm}$, 最高 $210.5 \mathrm{~mm}$, 最低 $82.9 \mathrm{~mm}$,多集中于 $7 、 8 、 9$ 三个月, 约占全年 $65 \%$ 。空气相对湿度 $46 \%$, 年蒸发量高达 $2390 \mathrm{~mm}$, 年平均气温 $7.6{ }^{\circ} \mathrm{C}$, 最高达 $39.1{ }^{\circ} \mathrm{C}$, 最低 为 $-27.3{ }^{\circ} \mathrm{C}$, 年 $\geqslant 10{ }^{\circ} \mathrm{C}$ 积温为 $3085^{\circ} \mathrm{C}$, 属干旱荒漠 气候。风向以西北风为主,年平均风速为 3.2 $\mathrm{m} \mathrm{s}^{-1}$ 最大风速 $21 \mathrm{~m} \mathrm{~s}^{-1}$, 年大风 $\left(\geqslant 17 \mathrm{~m} \mathrm{~s}^{-1}\right)$ 日 数平均为 $15 \mathrm{~d}$ 。地带性土壤为灰棕漠土。天然植被 以泡泡刺和红砂为主, 受风沙长期干扰, 泡泡刺呈簇 生状, 并有灌丛沙堆发育, 而红砂种群周围基本无灌 丛沙堆发育, 生长矮小。

\section{2 调查方法}

在野外踏查的基础上，根据植被分布、盖度、样 方大小所能包含的信息量，在研究区选择一块 500 $\mathrm{m} \times 500 \mathrm{~m}$ 受人为干扰较小的样地为研究对象。在 样地内采用规则的网格状布样方法 (Issaks \& Srivastava，1989) 将样地划分成 2500 个小样方 $(10 \mathrm{~m} \times 10$ $\mathrm{m})$ 样方间纵向和横向最小距离为 $10 \mathrm{~m}$, 最大距离 为 $500 \mathrm{~m}$ 。调查每个小样方内的植物种类、密度、盖 度以及泡泡刺灌丛沙堆的高度和体积 (表1)。其中 
表 1 样地内植被属性(均值 \pm 标准偏差)

Table 1 The characteristics of vegetation in the study area (Mean $\pm S D$ )

\begin{tabular}{lcc}
\hline & 泡泡刺 Nitraria sphaerocarpa & 红砂 Reaumuria soongorica \\
\hline 盖度 Coverage $(\%)$ & $1.56 \pm 2.34$ & $0.23 \pm 0.22$ \\
密度 Density (individuals $\left.10^{-2} \mathrm{~m}^{-2}\right)$ & $1.52 \pm 1.76$ & $2.34 \pm 2.18$ \\
灌丛沙堆高度 Height of shrub sand dune $(\mathrm{m})$ & $0.18 \pm 0.15$ & - \\
灌丛沙堆体积 Volume of shrub sand dune $\left(\mathrm{m}^{3}\right)$ & $0.10 \pm 0.18$ & - \\
\hline
\end{tabular}

盖度数据是通过测量每个样方内红砂和泡泡刺灌丛 的冠幅得到的, 因为样地内主要有泡泡刺和红砂两 种植物, 泡泡刺积沙形成灌丛状, 而红砂孤立分布, 以两种植物的冠幅所占整个样方的比例作为盖度 值。

本文采用 2500 个 $10 \mathrm{~m} \times 10 \mathrm{~m}$ 小样方中获取的 植物盖度数据来分析采样尺度和划区方式对荒漠植 被空间异质性的影响。选择盖度作为分析数据的原 因是荒漠植被的高度和密度在空间上差异不显著， 不能很好地反映植被空间格局, 而生物量在大面积 上调查存在较大的难度。盖度值不仅反映荒漠植物 的长势, 而且能明显地反映种群空间格局。另外, 本 文采用的种群盖度是实测值而不是估计值，因此采 用盖度数据可以反映种群空间异质性的尺度效应和 划区效应。

\section{3 数据分析方法}

\subsection{1 植被空间异质性分析}

首先对调查的每块样地植被盖度数据进行描述 统计分析, 在此基础上建立变异函数的理论模型 (Robertson, 1987)。确定变异函数的主要参数包括 块金值 $\left(C_{0}\right)$ 、基台值 $\left(C_{0}+C\right)$ 、变程 $\left(A_{0}\right)$ 和各向异性 比 $K(h)$ 等。根据变异函数理论, 随着间隔距离 $h$ 的增大 变异函数 $r(h)$ 从非零值达到一个相对稳定 的常数时,该常数称为基台值,当间隔距离 $h=0$ 时, $r(0)=C_{0}$, 该值称为块金值。基台值是系统或 系统属性中最大的变异, 变异函数 $r(h)$ 达到基台值 时的间隔距离 $A_{0}$ 称为变程 (王政权,1999)。其中块 金值表示随机部分的空间异质性，较大的块金值表 明较小尺度上的某种过程不可忽视。当样地面积过 小 就会出现纯块金效应, 从而不能真实地反映空间 异质性。基台值表示系统属性或区域变化量最大变 异, $C_{0}+C$ 越大表示总的空间异质性程度越高。 $A_{0}$ 表示空间相关尺度, 当采样间隔距离大于 $A_{0}$ 时, 变 量与空间不相关, 即变程是采样面积的临界尺度，小 于变程的采样尺度也不能真实地反映变量空间异质 性。变异函数采用下式计算 (Matheron, 1963)：

$$
r(h)=\frac{1}{2 N(h)} \sum_{i=1}^{n}\left[z\left(x_{i}\right)-z\left(x_{i+h}\right)\right]^{2}
$$

式中 : $r(h)$ 为变异函数 $z\left(x_{i}\right)$ 代表点 $x_{i}$ (样方) 的荒 漠植被盖度值, $z\left(x_{i+h}\right)$ 代表与 $x_{i}$ 间距为 $h$ 样点的 样方盖度值; $N(h)$ 是相距 $h$ 的所有点对的数目。 另外，各向异性比计算公式为：

$$
K(h)=r\left(h, \theta_{1}\right) / r\left(h, \theta_{2}\right)
$$

式中: $K(h)$ 为各向异性比, $r\left(h, \theta_{1}\right)$ 和 $r\left(h, \theta_{2}\right)$ 分别 为南北、东西两个方向上的变异函数。如果 $K(h)$ 等于或接近于 1 则空间异质性为各向同性, 否则为 各向异性。

\subsection{2 数据增聚方式与划区方案}

不同尺度上的盖度值是采用数据增聚方式获 得。尺度效应分析主要从两个方面出发 :一是保持 粒度 (样方) 不变, 增加幅度 (样地) 分析其对空间异 质性的影响;二是保持幅度不变, 改变粒度进行分 析。

首先按照第一种尺度变化，以调查的第一个样 方为基准点把整个样地 $(500 \mathrm{~m} \times 500 \mathrm{~m})$ 分成 $50 \mathrm{~m} \times$ $50 \mathrm{~m} 、 100 \mathrm{~m} \times 100 \mathrm{~m} 、 200 \mathrm{~m} \times 200 \mathrm{~m} 、 300 \mathrm{~m} \times 300 \mathrm{~m}$ 、 $400 \mathrm{~m} \times 400 \mathrm{~m}$ 和 $500 \mathrm{~m} \times 500 \mathrm{~m} 6$ 种不同幅度的样地 单元。再按照第二种尺度变化增加空间粒度, 即将 $10 \mathrm{~m} \times 10 \mathrm{~m}$ 的样方变成 $10 \mathrm{~m} \times 20 \mathrm{~m} 、 20 \mathrm{~m} \times 20 \mathrm{~m} 、 20$ $\mathrm{m} \times 30 \mathrm{~m} 、 30 \mathrm{~m} \times 30 \mathrm{~m} 、 30 \mathrm{~m} \times 40 \mathrm{~m} 、 40 \mathrm{~m} \times 40 \mathrm{~m} 、 40 \mathrm{~m}$ $\times 50 \mathrm{~m}$ 和 $50 \mathrm{~m} \times 50 \mathrm{~m}$ 的不同大小的粒度。然后用 地统计学中的变异函数理论模型计算各个尺度上的 空间异质性参数, 并以空间比较的方法分析尺度变 化对荒漠绿洲过渡带植被空间异质性的影响。

划区效应分析是在同一样地尺度 $(500 \mathrm{~m} \times 500$ $\mathrm{m})$ 上，以不同的划区方向和划区形状把整块样地数 据聚合成 $10 \mathrm{~m} \times 200 \mathrm{~m} 、 20 \mathrm{~m} \times 100 \mathrm{~m} 、 40 \mathrm{~m} \times 50 \mathrm{~m} 、 50$ $\mathrm{m} \times 40 \mathrm{~m} 、 100 \mathrm{~m} \times 20 \mathrm{~m}$ 和 $200 \mathrm{~m} \times 10 \mathrm{~m}$ 等不同形状 和不同方向的网格。用变异函数的理论模型对不同 划区方式的聚合数据进行分析，比较空间异质性参 数的变化，从而分析不同划区方式对异质性参数的 影响。 


\section{2 结果与讨论}

\section{1 植被空间异质性的尺度效应}

\subsection{1 幅度变化对植被空间异质性的影响}

图 1 和图 2 是按照数据增聚方式计算的空间异 质性参数变化过程, 从图中可以看出, 荒漠绿洲过渡 带植被空间异质性在不同尺度上的参数不尽相同。 当样地面积为 $50 \mathrm{~m} \times 50 \mathrm{~m}$, 样方总数为 25 个, 泡泡 刺和红砂种群的块金值与基台值之比接近 1 ,变异 函数出现纯块金效应, 预示着这两种种群在整个尺 度上具有恒定的变异。出现这种现象的原因在于： 一是样本数量太少, 满足不了地统计学计算变异函 数的要求：二是样地面积太小，包容不了影响植被空 间异质性的环境因子，所以在这个尺度 (样地面积) 上计算的空间异质性参数不能真实反映整个荒漠 绿洲过渡带的植被空间格局。当尺度(样地面积)
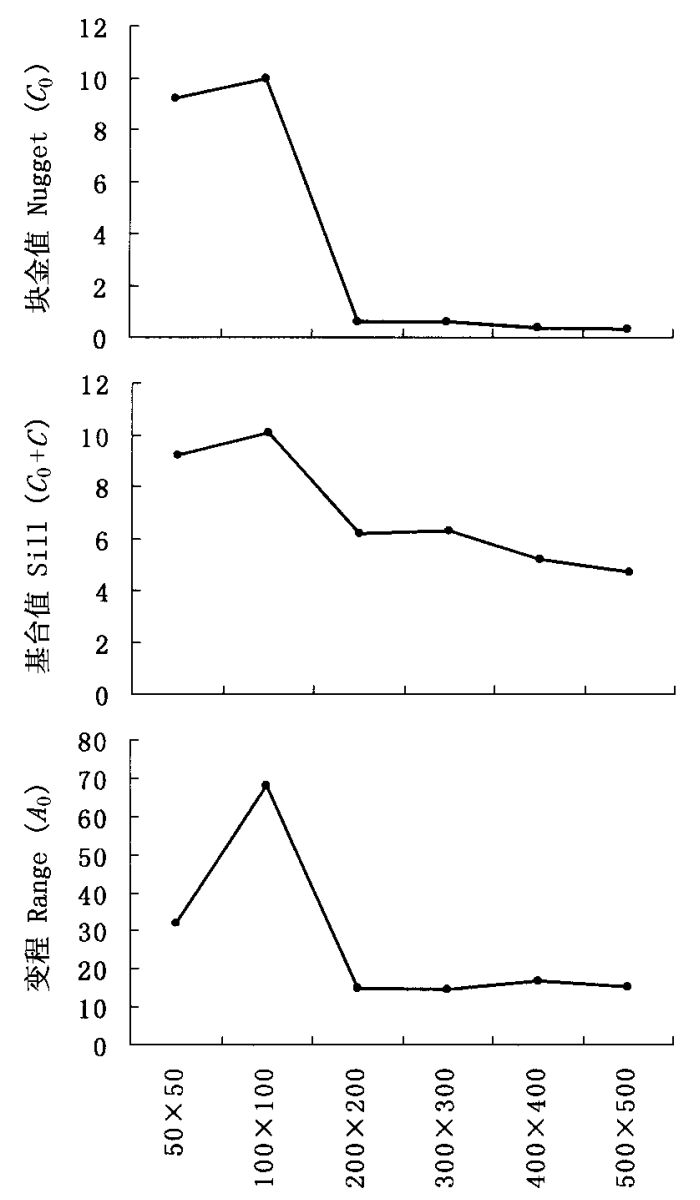

样地面积 Polt area $\left(\mathrm{m}^{2}\right)$

图 1 泡泡刺种群的空间异质性参数与样地面积的关系 Fig.1 The relationship between spatial heterogeneity parameters of Nitraria sphaerocarpa population and plot areas

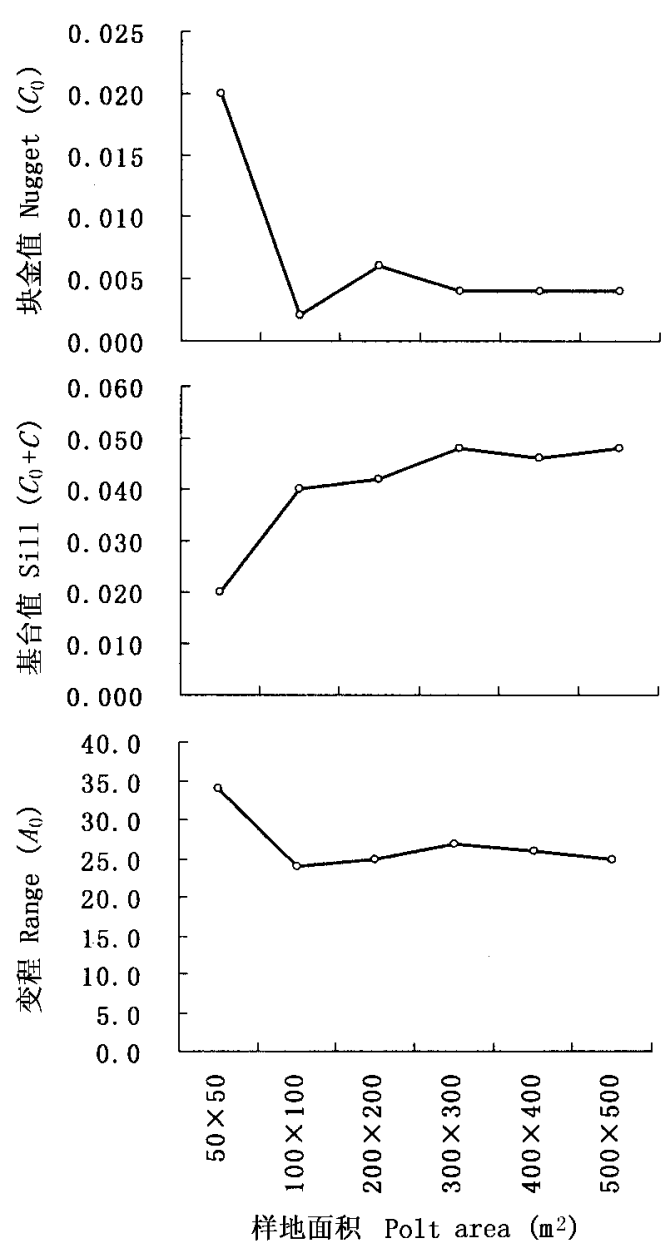

图 2 红砂种群的空间异质性参数与样地面积的关系

Fig.2 The relationship between spatial heterogeneity parameters of Reaumuria soongorica population and plot areas

在 $200 \mathrm{~m} \times 200 \mathrm{~m}$ 以上时, 空间异质性参数 $\left(C_{0} 、 C_{0}+\right.$ $C$ 和 $\left.A_{0}\right)$ 变异较小, 各个参数值基本稳定在一个较 小的范围内。表明反映荒漠绿洲过渡带植被空间异 质性所要调查的样地面积大小存在一个下限值, 当 低于该值时, 就不能真实地反映, 如果超过该值, 样 地面积的大小对空间异质性分析影响不大, 各个参 数变化稳定。这种稳定性可以看作是种群的内部因 素起作用，而与调查时所选的样地大小无关。另外， 不同种群的空间异质性受调查样地面积的影响程度 不同 样地面积大小的临界值也不同。泡泡刺对环 境因子的反应比红砂敏感, 空间依赖性强。所以反 映泡泡刺空间异质性的样地面积大小的临界值要比 红砂的大。从图 1 和图 2 可以看出, 调查泡泡刺种 群空间分布格局的样地面积应不小于 $200 \mathrm{~m} \times 200$ $\mathrm{m}$, 而红砂种群应不小于 $100 \mathrm{~m} \times 100 \mathrm{~m}$ 。当然样地 面积也不能过大, 否则一方面增加工作量, 另一方面 会造成跨群落调查而使情况复杂化 (朱锦悡等, 
1999)。

2.1.2 粒度变化对植被空间异质性的影响

从图 3 和图 4 可以看出, 基台值随调查样方增 大都呈降低的趋势, 而变程随样方增大呈上升的趋 势。另外, 泡泡刺盖度的块金值随样方面积增大变 异较大, 无明显的变化规律, 而红砂盖度的块金值随 样方面积增大呈幂函数下降趋势。表明泡泡刺的空 间异质性中随机因素对样方尺度比较敏感, 而且它 与空间尺度的依赖关系是难以预测的。其原因可能 是处在荒漠绿洲过渡带的泡泡刺种群分布受地表径 流、沙埋和地下水埋深等多因素影响, 在空间分布上 有较强的各向异性结构 (图 5)。两种种群盖度的变 程随样方尺度增大都呈上升趋势, 其原因是植物群 落随调查样方增大其样方间属性差异减小, 但是由 于调查样方增大 (因为在变异函数分析过程中一般 认为调查样方是同质的，样方尺度对应的是由随机 因素引起的异质性尺度) 使得由随机因素引起的空 间异质性尺度增大，因此，空间相关性对应的尺度
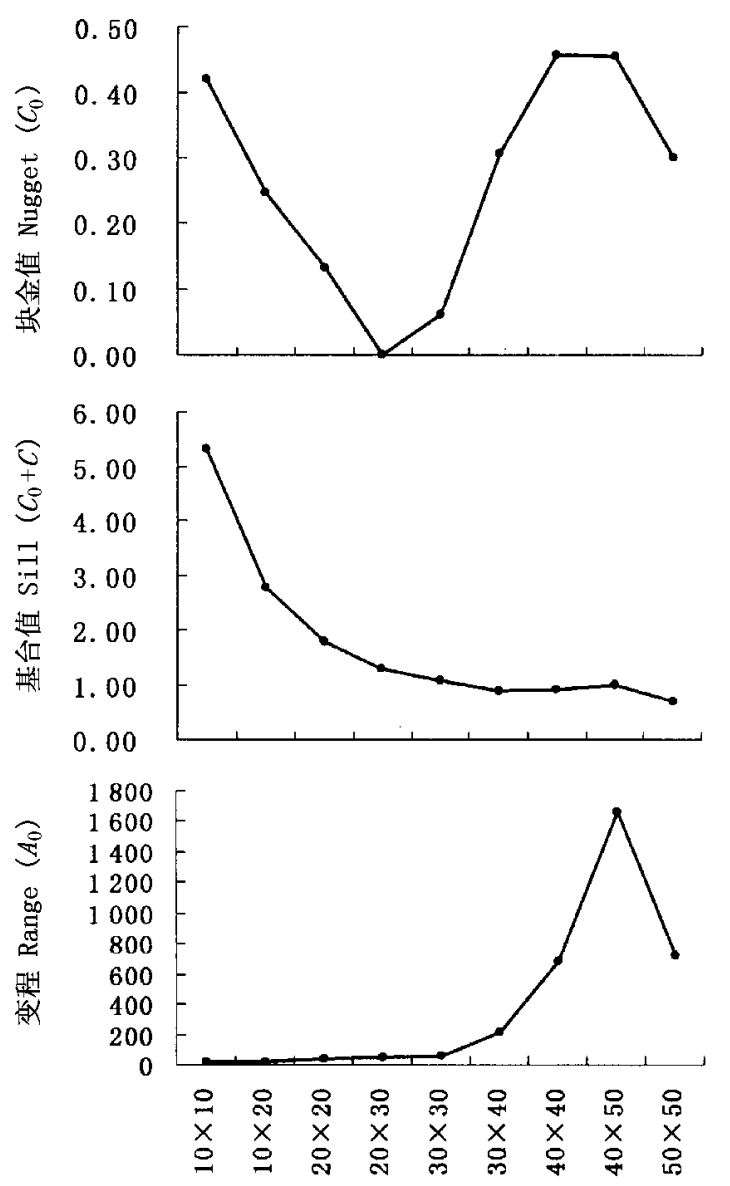

样方面积 Plot area $\left(\mathrm{m}^{2}\right)$

图 3 泡泡刺种群空间异质性参数与样方面积关系

Fig.3 The relationship between spatial heterogeneity parameters of Nitraria sphaerocarpa population and plot areas
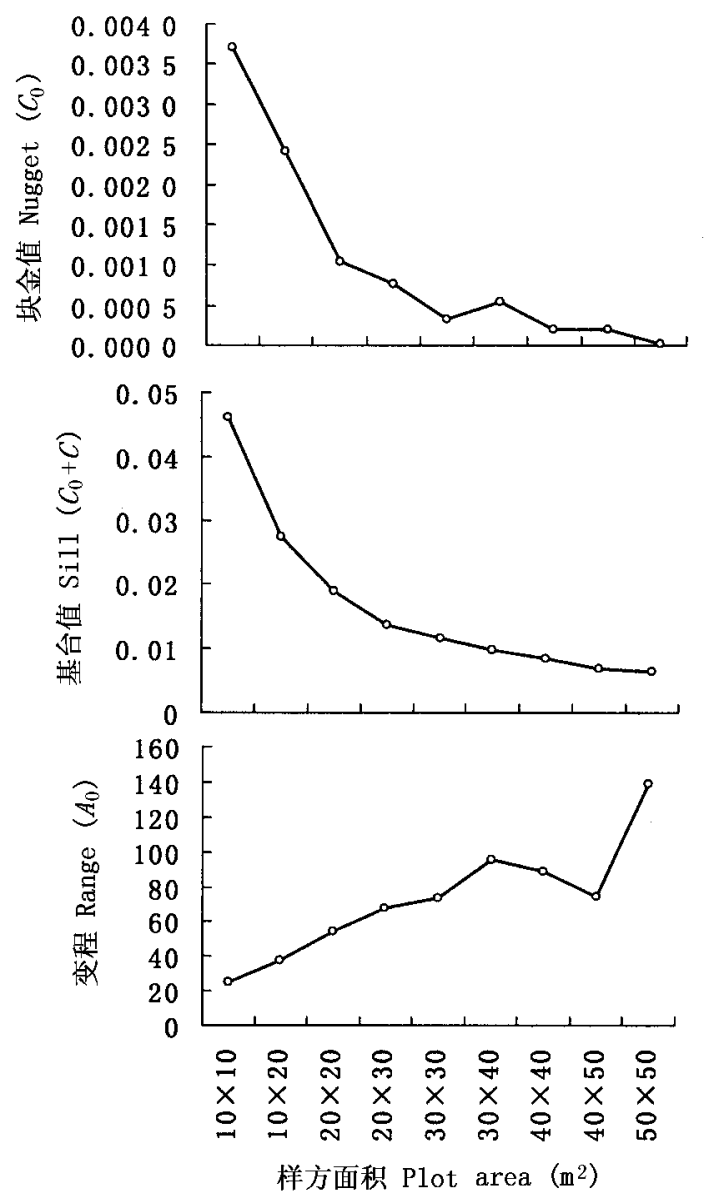

图 4 红砂种群空间异质性参数与样方面积关系

Fig.4 The relationship between spatial heterogeneity parameters of Reaumuria soongorica population and plot areas

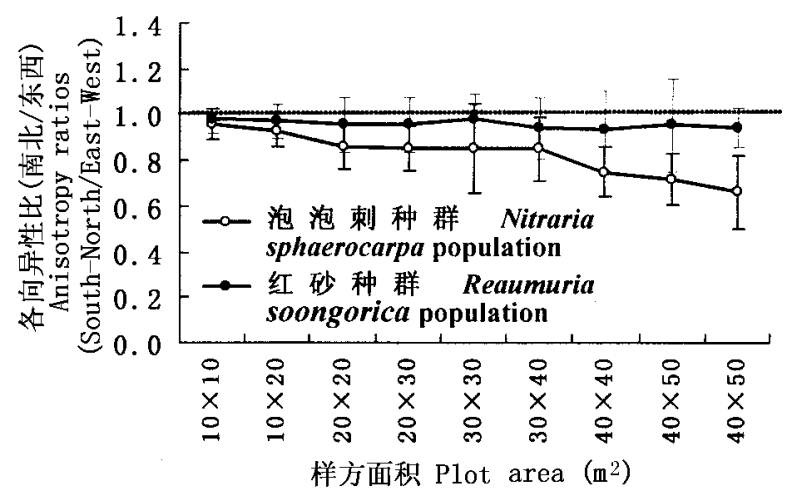

图 5 不同样方尺度下泡泡刺和红砂种群的各向异性结构

Fig.5 The anisotropy ratios of Nitraria sphaerocarpa population and Reaumuria soongorica population at different plot areas

也必然会增大。荒漠植被调查中当样地面积确定 后, 调查样方大小也不能随意选择，因为样方太小， 样方间的数据差异很大, 这种差异足以掩盖由空间 位置不同带来的差异(李友常等,1997);样方太大， 样方间的数据趋于同质, 异质性程度减小 结果难以 
反映植被空间异质性。此外，样方面积变化对泡泡 刺种群空间异质性的各向异性结构影响明显。从图 5 可以看出，红砂种群的各向异性结构对样方尺度 不敏感,在样方面积增大过程中，各向异性比(南北/ 东西)没有明显的变化。但是泡泡刺种群的各向异 性比(南北/东西)随样方面积增大呈逐渐减小趋势， 即各向异性比值逐渐偏离 1 ,各向异性结构越明显。 但是当样方面积在 $20 \mathrm{~m} \times 20 \mathrm{~m}$ 到 $30 \mathrm{~m} \times 40 \mathrm{~m}$ 范围 时, 泡泡刺种群的各向异性比值较稳定 $(0.859 \sim$ $0.852)$ 。以上分析可以得出, 泡泡刺和红砂种群的 空间异质性参数基台值在样方大于 $20 \mathrm{~m} \times 20 \mathrm{~m}$ 变 化相对缓和，而变程在样方 $10 \mathrm{~m} \times 10 \mathrm{~m}$ 到 $30 \mathrm{~m} \times 30$ $\mathrm{m}$ 内变化较稳定。故调查荒漠绿洲过渡带上泡泡刺
和红砂种群的空间格局时，样方面积在 $20 \mathrm{~m} \times 20 \mathrm{~m}$ 到 $30 \mathrm{~m} \times 30 \mathrm{~m}$ 较为合适。

\section{2 植被空间异质性的划区效应}

由表 2 可见, 荒漠绿洲过渡带植被空间异质性 参数对划区方式的敏感性较强, 尤其是块金值和变 程, 因划区方式不同而发生剧烈变化, 变异系数为 $0.982 \sim 1.565$ 。对划区效应敏感性也因植物种群而 异 如泡泡刺种群的空间异质性参数对划区方式的 敏感性明显高于红砂, 可能是泡泡刺种群的各向异 性高于红砂种群(图 5), 在空间分布上受地表径流、 风沙危害等的影响更强烈, 而红砂分布相对均匀。 总之 划区方式对分析荒漠绿洲过渡带植被空间异 质性的影响是显著的。

表 2 不同划区方式泡泡刺和红砂种群空间异质性参数

Table 2 The variance of spatial heterogeneity parameters of Nitraria sphaerocarpa population and Reaumuria soongorica population for different zoning approaches

\begin{tabular}{|c|c|c|c|c|c|c|c|c|}
\hline \multirow{2}{*}{$\begin{array}{c}\text { 划区方式 } \\
\text { Zoning approach }\end{array}$} & \multicolumn{4}{|c|}{ 泡泡刺种群 Nitraria sphaerocarpa } & \multicolumn{4}{|c|}{ 红砂种群 Reaumuria soongorica } \\
\hline & 模型 Model & $C_{0}$ & $C_{0}+C$ & $A_{0}(\mathrm{~m})$ & 模型 Model & $C_{0}$ & $C_{0}+C$ & $A_{0}(\mathrm{~m})$ \\
\hline $10 \mathrm{~m} \times 200 \mathrm{~m}$ & 指数 Exponent & 0.004 & 0.170 & 4 & 指数 Exponent & 0.0019 & 0.0042 & 137.5 \\
\hline $20 \mathrm{~m} \times 100 \mathrm{~m}$ & 指数 Exponent & 0.096 & 0.741 & 8.6 & 指数 Exponent & 0.0005 & 0.0073 & 21.2 \\
\hline $40 \mathrm{~m} \times 50 \mathrm{~m}$ & 指数 Exponent & 0.454 & 0.978 & 554.2 & 指数 Exponent & 0.0002 & 0.0068 & 24.8 \\
\hline $50 \mathrm{~m} \times 40 \mathrm{~m}$ & 指数 Exponent & 0.001 & 0.644 & 63.5 & 指数 Exponent & 0.0001 & 0.0069 & 40.6 \\
\hline $100 \mathrm{~m} \times 20 \mathrm{~m}$ & 指数 Exponent & 0.204 & 0.461 & 184 & 指数 Exponent & 0.0007 & 0.0066 & 33.8 \\
\hline $200 \mathrm{~m} \times 10 \mathrm{~m}$ & 指数 Exponent & 0.098 & 0.764 & 10.9 & 指数 Exponent & 0.0005 & 0.0064 & 19.1 \\
\hline 变异系数 $C V$ & - & 1.188 & 0.447 & 1.565 & - & 0.9820 & 0.1750 & 0.985 \\
\hline
\end{tabular}

3 如何解决植被空间异质性的可塑性面积 单元问题

目前关于如何解决可塑性面积单元问题, 大致 有 5 种途径 : 基本实体途径 (Basic entity approach)、最 优划区途径 (Optimal zoning approach)、敏感性分析途 径 (Sensitivity analysis approach)、摒旧创新途径和强 调所研究变量的变化速率 (Jelinski \& Wu, 1996)。 但是每种途径都存在不足, 如基本实体途径尽管能 避免可塑性面积单元问题, 但是在生态学或地理学 研究中, 并非总能说明什么是基本实体, 例如, 密度、 通量、覆盖度等变量均是与面积有关的。对我们所 研究的荒漠绿洲过渡带植被空间格局采取敏感性分 析途径来解决可塑性面积单元问题是可行的，这个 途径是通过一系列研究来确定或大致掌握可塑性面 积单元影响的范围和强度 (Fotheringham , 1989)。它 可以回答哪些变量对尺度效应最为敏感? 哪些变量 对尺度最不敏感? 哪些变量是可预测的? 哪些变量 是不可预测的? 尽管敏感性分析比较繁琐, 但它毕 竟是一种有效的途径。
采用不同尺度和划区方式对泡泡刺和红砂种群 的空间异质性分析表明: 空间异质性参数块金值、基 台值和变程对空间尺度比较敏感, 因尺度变化均有 较大的变异, 并且不同参数的敏感尺度范围不同 如 块金值和基台值在小尺度 (包括幅度和粒度)上比较 敏感, 变异最大, 随尺度增大时变化稳定。而变程在 小尺度(粒度)上变化相对稳定, 随粒度增大其变化 剧烈，对尺度的敏感性也增强。另外块金值和变程 对划区效应的敏感程度高，而基台值对划区效应的 敏感性相对较弱。

\section{4 结 论}

对于荒漠绿洲过渡带植被来说, 不同的空间异 质性参数对可塑性面积单元 (尺度和区划方式)的敏 感程度和敏感范围不同。本研究表明, 可以通过确 定这些参数的敏感程度和范围来减弱可塑性面积单 元的影响。另外, 空间异质性程度越高的种群对可 塑性面积单元越敏感，对荒漠绿洲过渡带泡泡刺和 红砂种群的例证研究表明: 分析泡泡刺种群空间异 
质性时, 应采用不小于 $200 \mathrm{~m} \times 200 \mathrm{~m}$ 的样地面积, $20 \mathrm{~m} \times 20 \mathrm{~m}$ 到 $30 \mathrm{~m} \times 30 \mathrm{~m}$ 的样方尺度较为合适。 红砂种群应采用不小于 $100 \mathrm{~m} \times 100 \mathrm{~m}$ 的样地面积， $20 \mathrm{~m} \times 20 \mathrm{~m}$ 到 $30 \mathrm{~m} \times 30 \mathrm{~m}$ 的样方尺度。

\section{参 考 文 献}

Chen, Y. F. (陈玉福) \& M. Dong (董鸣). 2003. Spatial heterogeneity in ecological systems. Acta Ecologica Sinica (生态学 报), 23: $346 \sim 352$. ( in Chinese with English abstract)

Dudley, G. 1991. Scale, aggregation, and the modifiable area unit problem. The Operational Geographer, 9: $28 \sim 33$.

Fotheringham, S. 1989. Scale-independent spatial analysis. In: Goodchild, M. F. \& S. Gopal eds. Accuracy of spatial databases. London: Taylor and Francis. $221 \sim 228$.

Gallagher, R. B., J. Fischman \& P. J. Hines. 1995. Big questions for a small planet. Science, 269: 283.

Gehlke, C. E. \& H. Biehl. 1934. Certain effects of grouping upon the size of the correlation coefficient in census tract material. Journal of American Statistical Association, 29 (Suppl.) : 169 170 .

Hay, G. J., T. Blaschke, D. J. Marceau \& A. Bouchard. 2003. A comparison of three image-object methods for the multiscale analysis of landscape structure. Journal of Photogrammetry \& Remote Sensing, 57: $327 \sim 345$.

Issaks, E. H. \& R. M. Srivastava. 1989. An introduction to applied geostatistics. New York: Oxford University Press. $68 \sim 97$.

Jelinski, D. E. \& J. Wu. 1996. The modifiable areal unit problem and implication for landscape ecology. Landscape Ecology, 11: $129 \sim 140$.

Jenerette, G. D. \& J. G. Wu. 2000. On the definitions of scale. Bulletin of the Ecological Society of America, 81: $104 \sim 105$.

Levin, S. A. 1992. The problem of pattern and scale in ecology. Ecology, 73: $1943 \sim 1967$.

Li, Y. C. (李友常), N. B. Xia (夏乃斌), Q. H. Tu (屠泉 洪), Y. Q. Luo (骆有庆) \& J. B. Wen (温俊宝). 1997. A geostatistical analysis on spatial patterns of Anoplophora glabripennis in poplars. Acta Ecologica Sinica (生态学报), 17: $393 \sim 401$. (in Chinese with English abstract)

Matheron, G. 1963. Principles of geostatistics. Economic Geology, 58: $1246 \sim 1266$.

Openshaw, S. \& P. Taylor. 1979. A million or so correlation coefficients: three experiments on the modifiable areal unit problem. In: Wrigley, N. ed. Statistical application in the spatial sciences. London: Qion. $127 \sim 144$.

Robertson, G. P. 1987. Geostatistics in ecology: interpolating with known variance. Ecology, 68: $744 \sim 748$.

Turner, M. G. 1989a. Effects of changing spatial scale on the analysis of landscape pattern. Landscape Ecology, 3: 153 162 .

Turner, M. G. 1989b. Landscape ecology: the effect of pattern on process. Annual Review of Ecology and Systematics, 20: $171 \sim$ 197.

Wang, Z. Q. (王政权), Q. C. Wang (王庆成) \& H. B. Li (李哈滨). 2000. Characteristics and comparison of spatial heterogeneity of the main species of Korean pine in old growth forests. Acta Phytoecologica Sinica (植物生态学报), 24: 718 723. ( in Chinese with English abstract)

Wang, Z. Q. (王政权). 1999. Geostatistics and its application in ecology. Beijing: Science Press. 150 156. (in Chinese)

Wu, J. G. \& Y. Qi. 2000. Dealing with scale in landscape analysis: an overview. Geographic Information Sciences, 6: $1 \sim 5$.

Wu，J. G. (邬建国). 1996. Paradigm shift in ecology: an overview. Acta Ecologica Sinica (生态学报), 16: $449 \sim 460$. (in Chinese with English abstract)

Wu, J. G. (邬建国). 2000. Landscape ecology: pattern, process, scale and hierarchy. Beijing: Higher Education Press. 142 $\sim 152$. (in Chinese)

Yule, G. \& M. Kendall. 1950. An introduction to the theory of statistics. New York: Charles Griffin and Company Limited.

Zhu, J. M. (朱锦檚) \& Z. L. Jiang (姜志林). 1999. The modifiable areal unit problem of species diversity in the forest community of northern Fujian Province. Acta Ecologica Sinica (生态学 报), 19: 304 311. (in Chinese with English abstract)

责任编委 : 王政权 责任编辑 :刘丽娟 